\title{
Management of Diarrhea in Patients with HER2- Positive Breast Cancer Treated with Neratinib: A Case Series and Summary of the Literature
}

\author{
Megan L. Kruse · Irene M. Kang • Nusayba A. Bagegni • \\ W. Todd Howell • Halle C. F. Moore • Cynthia H. Bedell • \\ Christopher T. Stokoe
}

Received: September 13, 2021 / Accepted: October 29, 2021 / Published online: November 20, 2021

(c) The Author(s) 2021

\begin{abstract}
Introduction: Neratinib and neratinib-based combinations have demonstrated efficacy for treatment of human epidermal growth factor receptor 2-positive (HER2+) early-stage and metastatic breast cancers. However, diarrhea has been reported as a common adverse event leading to neratinib discontinuation. Results from the CONTROL trial suggest that proactive diarrhea management with antidiarrheal prophylaxis or dose escalation of neratinib from a lower starting dose to the full FDA-approved
\end{abstract}

\footnotetext{
M. L. Kruse (ه) · H. C. F. Moore

Division of Hematology and Medical Oncology, Taussig Cancer Institute, Cleveland Clinic, 9500 Euclid Avenue, CA-60, Cleveland, OH 44195, USA e-mail: krusem@ccf.org

I. M. Kang

Norris Comprehensive Cancer Center, Keck School of Medicine, University of Southern California, Los Angeles, CA, USA

N. A. Bagegni

Division of Medical Oncology, Alvin J. Siteman Cancer Center, Washington University in St. Louis School of Medicine, St. Louis, MO, USA

W. T. Howell

Pharmacy, Hematology and Oncology Associates of Alabama, Birmingham, AL, USA

C. H. Bedell · C. T. Stokoe

Texas Oncology, Plano East Cancer Center, Plano, TX, USA
}

dose of $240 \mathrm{mg} /$ day can reduce the incidence, duration, and severity of neratinib-associated diarrhea in patients with early-stage breast cancer. Dose escalation has been included in the FDA-approved label for both early-stage and metastatic HER2+ breast cancer since June 2021.

Case series: This series of five cases details realworld clinical implementation of strategies for management of neratinib-induced diarrhea in patients with early-stage and metastatic HER2 + breast cancer, including a patient with a pre-existing gastrointestinal disorder.

Management and outcome: In four of five cases, diarrhea was managed with neratinib dose escalation, and antidiarrheal prophylaxis with loperamide plus colestipol was used in the remaining case. Management of diarrhea allowed all patients to remain on therapy.

Discussion: This case series shows that neratinib-associated diarrhea can be managed effectively with neratinib dose escalation from a lower initial starting dose and/or prophylactic antidiarrheal medications in a real-world clinical setting. The findings highlight the importance of patient-provider communication in proactive management of adverse events. Widespread implementation of the strategies described here may improve adherence and thereby clinical outcomes for patients with HER2+ breast cancer treated with neratinib. 
Keywords: Diarrhea; Early-stage breast cancer; HER2; Metastatic breast cancer; Neratinib

\section{Key Summary Points}

Neratinib and neratinib-based combinations have demonstrated efficacy for treatment of human epidermal growth factor receptor 2-positive (HER2+) earlystage and metastatic breast cancers.

Proactive management may reduce the incidence, duration, and severity of diarrhea, a common adverse event reported with neratinib, allowing patients to remain on treatment.

This series of five cases details real-world clinical implementation of strategies for management of neratinib-induced diarrhea in patients with early-stage and metastatic HER2+ breast cancer, including a patient with a pre-existing gastrointestinal disorder.

These cases show that neratinib-associated diarrhea can be managed effectively with neratinib dose escalation and/or prophylactic antidiarrheal medications and highlight the importance of patientprovider communication in proactive management.

Widespread implementation of the strategies described in this case series may improve adherence to therapy and thereby clinical outcomes for patients with HER2+ breast cancer treated with neratinib.

\section{INTRODUCTION}

Breast cancers characterized by human epidermal growth factor receptor 2 (HER2) overexpression represent a common, aggressive subtype, comprising approximately $20 \%$ of breast cancers [1]. Although trastuzumab has improved outcomes for patients with HER2- positive (HER2+) breast cancer, long-term follow-up of phase 3 trials has demonstrated that cancer recurs within 10 years in $25-31 \%$ of patients treated with adjuvant trastuzumab [1-4]. Metastatic and recurrent HER2+ breast cancers are often associated with an increased propensity for developing central nervous system (CNS) metastases, which occur in up to $50 \%$ of patients with HER $2+$ metastatic breast cancer (MBC) $[1,5,6]$.

In recent years, novel neoadjuvant, adjuvant, and extended adjuvant regimens have reduced the risk of recurrence for patients with HER2 + early-stage breast cancer (ESBC) [1]. The phase 3 NOAH study demonstrated that addition of trastuzumab to neoadjuvant chemotherapy was associated with a greater proportion of patients achieving a pathologic complete response (pCR) and a significant improvement in event-free survival [7]. pCR rate was further improved with the use of neoadjuvant dual HER2 blockade with trastuzumab and pertuzumab based on the results of the phase 2 NeoSphere and TRYPHAENA trials $[8,9]$. The combination of trastuzumab and pertuzumab showed efficacy in the phase 3 APHINITY trial, and results of the phase 3 KATHERINE trial have supported adjuvant adotrastuzumab emtansine (T-DM1) as an option for patients with residual disease [10, 11]. In patients with HER2+ ESBC treated with adjuvant trastuzumab-based therapy, extended adjuvant therapy with the oral, irreversible panHER tyrosine kinase inhibitor (TKI) neratinib significantly improved invasive disease-free survival in the phase 3 ExteNET study $[12,13]$.

HER2-targeted agents, including neratinib, have also improved outcomes for patients with HER2+ MBC. Regimens demonstrating efficacy in this setting include pertuzumab in combination with trastuzumab and docetaxel, T-DM1, trastuzumab deruxtecan, and other trastuzumab plus chemotherapy combinations [1, 14-17]. Neratinib and other TKIs have shown efficacy in MBC in combination with trastuzumab, capecitabine, and/or other cytotoxic therapies [18-26]. In addition, TKI-containing combinations, such as neratinib in combination with capecitabine or paclitaxel, lapatinib plus capecitabine, and tucatinib plus 
trastuzumab and capecitabine, have shown benefit in patients with CNS metastases [18, 27-30].

Neratinib is approved for use as extended adjuvant therapy for HER2+ ESBC or in combination with capecitabine in the treatment of HER2+ MBC [31]. In the current National Comprehensive Cancer Network Guidelines, neratinib is included as a category $2 \mathrm{~A}$ recommendation for both HR+, HER2+ ESBC following adjuvant trastuzumab-containing therapy and HER2+ MBC third line and beyond and is also included as a treatment option for recurrent HER2+ breast cancer CNS metastases $[32,33]$. Gastrointestinal (GI)-related adverse events, especially diarrhea, are common with neratinib and can lead to early discontinuation. This is likely a class effect for TKIs with broader target profiles. The mechanism is not completely understood, but may involve dysregulated ion transport, inflammation, and mucosal injury $[12,13,18,23,24,26,34]$. In the ExteNET trial, in which the use of anti-diarrheal prophylaxis was not mandated, $95 \%$ of patients on extended adjuvant neratinib experienced diarrhea, including grade 3 diarrhea in $40 \%$ of patients. Twenty-eight percent of patients discontinued because of any treatment-emergent adverse event (TEAE) and 17\% discontinued because of diarrhea. [12, 35]. Early discontinuation is thought to negatively affect treatment outcomes, as subgroup analyses of ExteNET have shown that remaining on treatment for $\geq 11$ of the recommended 12 months is important for neratinib efficacy $[36,37]$. In the phase 3 NALA study of capecitabine plus either neratinib or lapatinib in patients with $\mathrm{MBC}$ previously treated with $\geq 2$ HER2-directed regimens, diarrhea was managed with mandatory loperamide prophylaxis in the neratinib plus capecitabine arm, in which $24.4 \%$ of patients experienced grade 3 diarrhea, resulting in discontinuation of neratinib in only $2.6 \%$ of patients [26].

The phase 2 CONTROL study was designed to prospectively evaluate strategies for managing neratinib-induced diarrhea in HER2+ ESBC [38]. CONTROL evaluated prophylactic use of various antidiarrheal medications as well as dose escalation (DE) of neratinib from a lower starting dose to the full US Food and Drug Administration (FDA)-approved dose of $240 \mathrm{mg} /$ day [38]. All preventive strategies reduced the incidence, duration, and severity of neratinib-induced diarrhea, lowering neratinib discontinuation rates compared to ExteNET, except in the loperamide-alone arm. Neratinib DE (2-week DE schedule: week $1=120 \mathrm{mg}$ /day, week $2=160 \mathrm{mg} / \mathrm{day}$, week 3 and onwards: $240 \mathrm{mg}$ /day) was identified as a particularly beneficial strategy for maintaining patients on therapy based on a $13 \%$ rate of grade $\geq 3$ diarrhea, a $3.3 \%$ rate of diarrhea-related discontinuation, and a $13.3 \%$ rate of discontinuation due to any TEAE [31, 38, 39]. A 2-week DE schedule also lowered the rate of constipation (all grades) compared with antidiarrheal strategies that mandated loperamide prophylaxis (33\% vs. $57-75 \%)$ [38].

To better understand clinical management of neratinib-associated diarrhea, this series describes four patients with ESBC and one patient with MBC treated with neratinib. The aim is to discuss strategies for managing neratinib-associated diarrhea in a real-world setting, with the goal of improving the patient experience and maintaining patients on therapy. These cases were identified through the authors' clinical practice. All identifying details, including the specific institutional settings, have been deidentified to protect the anonymity of the patients.

This article is based on previously conducted studies and anonymized case descriptions and does not contain any new studies with human participants or animals performed by any of the authors.

\section{CASE SERIES}

\section{Case 1}

The patient is a 40-year-old female teacher with no medical comorbidities who was diagnosed with stage III (cT3N1M0) infiltrating ductal carcinoma (IDC) of the right breast. The patient initially self-palpated a breast mass, delaying medical attention by 2.5 months because of lack of health insurance. Imaging revealed a $7-\mathrm{cm}$ 
breast mass and two abnormal lymph nodes. Biopsy confirmed estrogen receptor (ER)-positive $(+)$, progesterone receptor $(\mathrm{PR})$-negative (-), HER2+ IDC. She had a strong family history of multiple relatives with breast cancer, sarcoma, and CNS tumors, and genetic testing revealed a pathogenic heterozygous TP53 916C > T germline mutation consistent with $\mathrm{Li}-$ Fraumeni syndrome [40].

After a favorable clinical response to neoadjuvant therapy with TCHP (docetaxel, carboplatin, trastuzumab, and pertuzumab) for six cycles, the patient underwent mastectomy with immediate reconstruction. Surgical pathology revealed a pCR (ypTONO). As a result of toxicity associated with TCHP, she required antidiarrheal agents for grade 1 diarrhea. She completed 11 cycles of adjuvant trastuzumab plus pertuzumab and continues anastrozole, goserelin, and zoledronic acid.

Approximately 6.5 months after completion of adjuvant anti-HER2 therapy, she began extended adjuvant therapy with neratinib, which is ongoing. Neratinib was initiated with $\mathrm{DE}$ with a starting dose of $160 \mathrm{mg} /$ day, increasing by $40 \mathrm{mg}$ every two weeks (q2w) until reaching the recommended dose of $240 \mathrm{mg} /$ day. To manage diarrhea, the patient takes a maximum of 2 to $4 \mathrm{mg}$ loperamide daily (qd) as needed (PRN). The patient was seen weekly at neratinib initiation, then $\mathrm{q} 2 \mathrm{w}$, and now monthly. She is tolerating therapy well, with one or two soft-formed bowel movements per day, no nausea or vomiting, and no clinical signs of recurrence.

\section{Case 2}

The patient is a 62-year-old man, retired after a career in the military, who developed stage I (cT1N1M0) IDC of the right breast. He is receiving active therapy for multiple comorbidities, including diabetes, chronic obstructive pulmonary disease, atrial fibrillation, seizure disorder, and hypertension. The patient presented with a breast mass in the upper outer right quadrant; biopsy revealed $\mathrm{ER}+/ \mathrm{PR}+/$ HER2+ IDC. His family history includes one second-degree relative with breast cancer.
Following neoadjuvant therapy with four cycles of doxorubicin and cyclophosphamide (AC), followed by 12 weekly cycles of docetaxel plus trastuzumab, the patient underwent mastectomy and axillary nodal excision of a $3.9-\mathrm{cm}$ mass. The patient continued adjuvant trastuzumab to complete 1 year in total. Daily letrozole was added 7 months after trastuzumab initiation and is ongoing.

One week after completion of trastuzumab, the patient initiated extended adjuvant therapy with neratinib, ongoing for almost 1 year. Neratinib was initiated using DE combined with colestipol $2 \mathrm{~g}$ qd and loperamide PRN (maximum dose: $12 \mathrm{mg} \mathrm{qd}$ ). The patient started on $120 \mathrm{mg} /$ day neratinib, and the dose was increased by $40 \mathrm{mg} \mathrm{q} 2 \mathrm{w}$. The patient reached the recommended dose of $240 \mathrm{mg} /$ day with no toxicities. Due to loose stools in the absence of prophylaxis, he continues to take colestipol $2 \mathrm{~g}$ qd and loperamide PRN for diarrhea and is doing well. Because the patient is enrolled in a patient management program through a specialty pharmacy, he was assessed and counseled by the pharmacist at neratinib initiation, with follow-up twice in the first month of therapy and monthly thereafter.

\section{Case 3}

The patient is a 60-year-old female flight attendant diagnosed with stage IIIC (cT1N3M0) $\mathrm{ER}+/ \mathrm{PR}+/ \mathrm{HER} 2+\mathrm{IDC}$ of the right breast. She has no family history of breast cancer. Comorbidities include hyperlipidemia and hypertension, which are unmedicated but stable. The patient underwent lumpectomy to remove a $1.2-\mathrm{cm}$ tumor. Axillary lymph node dissection revealed 16 of 17 sampled lymph nodes involved with metastatic carcinoma.

The patient received adjuvant AC for 4 cycles, followed by 12 cycles of weekly paclitaxel with concurrent trastuzumab plus pertuzumab, with continuation of trastuzumab and pertuzumab to complete 1 year. After chemotherapy completion, the patient underwent radiation, followed by anastrozole, which is ongoing. 
Extended adjuvant therapy with neratinib was initiated $<1$ month after completion of trastuzumab-based therapy. The patient initiated neratinib on a DE protocol, with a starting dose of $120 \mathrm{mg} /$ day, combined with colestipol $1 \mathrm{~g}$ twice daily (bid), loperamide $4 \mathrm{mg}$ every $4 \mathrm{~h}$ PRN, and diphenoxylate hydrochloride $5 \mathrm{mg} /$ atropine sulfate $0.05 \mathrm{mg}$ every $6 \mathrm{~h}$ PRN. The neratinib dose was increased by $40 \mathrm{mg}$ q2w as tolerated by the patient. The patient experienced intermittent grade 2 diarrhea, which was controlled with this antidiarrheal regimen. Neratinib was paused for bilateral mastectomy, after which DE was reinitiated as before. The patient was unable to escalate to $240 \mathrm{mg}$ /day because of development of grade 2 to 3 diarrhea and instead maintained a 3 -day repeating dose pattern of 200, 240, and $240 \mathrm{mg}$ /day. Approximately 10 months into therapy, diarrhea had improved to the point that the patient stopped taking colestipol and managed any residual diarrhea with loperamide and diphenoxylate/ atropine PRN. The patient chose to remain on extended neratinib therapy for longer than 1 calendar year because of the interruption due to surgery.

\section{Case 4}

The patient is a 38-year-old woman employed in sales who developed stage II (cT2NOM0) ER+/ PR-/HER2+ IDC of the right breast. She is a nonsmoker and exercises regularly. She was diagnosed with Crohn's disease in the remote past, and her symptoms are managed with hyoscyamine and FODMAP (fermentable oligosaccharides, disaccharides, monosaccharides, and polyols) diet. She has no other comorbidities. Her family history includes a first-degree relative with breast cancer; genetic testing was negative for heritable mutations.

The patient underwent lumpectomy and sentinel lymph node biopsy, with no involvement of four sampled axillary lymph nodes. She received adjuvant $\mathrm{TCH}$ (docetaxel, carboplatin plus trastuzumab) for six cycles followed by trastuzumab to complete 1 year in total. Approximately 1 year after trastuzumab completion, the patient initiated neratinib for 12 months, along with ongoing tamoxifen.

Despite a history of Crohn's disease, the patient initiated neratinib at the full $240 \mathrm{mg} /$ day dose with prophylactic loperamide $4 \mathrm{mg}$ three times daily (tid) and colestipol $2 \mathrm{~g}$ bid. The patient experienced diarrhea for the first 1 to two weeks of treatment, including 1 day at grade 3 , but was then able to taper off antidiarrheal medications. In months 7 to 9 , she developed grade 2 palmar/plantar erythrodysesthesia (PPE), requiring neratinib dose reduction to $200 \mathrm{mg} /$ day at month 8 and management with emollient and triamcinolone cream. She was able to complete 12 months of therapy at the reduced dose.

\section{Case 5}

The patient is a 60-year-old female cafeteria worker who was originally diagnosed with stage II (cT1N1M0) ER-/PR+/HER2+ IDC of the right breast in 2004 and developed metastatic HER2 + recurrence involving bone and lymph nodes 4 years later. She has no comorbidities and no known family history of breast cancer. Initial treatment for stage II disease was right modified radical mastectomy followed by six cycles of adjuvant TAC (docetaxel, doxorubicin, and cyclophosphamide).

The patient has received multiple lines of therapy for $\mathrm{MBC}$, including vinorelbine plus trastuzumab at initial recurrence. After development of lung metastases, she was treated with T-DM1 on a clinical trial and experienced 6 years of disease control. She again experienced excellent disease control for 3 years with capecitabine plus lapatinib followed by slow progression of pulmonary metastases.

The patient was reluctant to change to an intravenous regimen and remained asymptomatic from her disease. Upon FDA approval of neratinib in combination with capecitabine for HER2 + MBC, lapatinib was replaced with neratinib $240 \mathrm{mg}$ qd with continuation of the same dose of capecitabine (1000 mg bid) based on prior tolerability. The patient received prophylactic loperamide $4 \mathrm{mg}$ tid but paused the regimen after 4 days because of grade 2 nausea and 
grade 1 to 2 constipation. She then reinitiated neratinib with a DE schedule, starting at $120 \mathrm{mg} /$ day with prophylactic loperamide $4 \mathrm{mg}$ bid, with improved tolerability. She was able to titrate the neratinib dose to $160 \mathrm{mg} /$ day after 1 week, to $200 \mathrm{mg} /$ day after 2 additional weeks, and to $240 \mathrm{mg} /$ day by week 5 . The patient was able to continue on therapy with loperamide bid with regular bowel movements and no further nausea. Three months into treatment with neratinib plus capecitabine, the patient had a near-complete radiographic response, with residual scarring in the areas of prior pulmonary nodules. She remains free of recurrent diarrhea.

\section{DISCUSSION}

The patient cases presented in this series provide examples of proactive management of diarrhea in patients with HER2 + ESBC and MBC treated with neratinib. In four of five cases, diarrhea was managed with neratinib DE, and antidiarrheal prophylaxis with loperamide plus colestipol was used in the remaining case. Management of diarrhea allowed all patients to remain on therapy, although two patients were unable to reach or maintain the full approved dose, in one case because of diarrhea and the other because of PPE.

Management of diarrhea with neratinib DE and antidiarrheal prophylaxis in this series is consistent with results from the CONTROL trial [38]. Neratinib DE was effective for managing and possibly reducing neratinib-associated early-onset diarrhea in cases 1, 2, 3, and 5, allowing therapy continuation. Three patients reached the full dose, and one patient was able to titrate to her maximum tolerable dose. DE was more effective for diarrhea management than loperamide prophylaxis in case 5 , consistent with results from CONTROL [38], in which antidiarrheal prophylaxis with loperamide alone was not as successful as neratinib DE. Results from CONTROL showed that 2-week neratinib DE with loperamide PRN was associated with the lowest rate of both diarrhea-associated discontinuation (3.3\% vs. $4-20 \%)$ and all-grade constipation (33\% vs. $57-75 \%$ in prophylaxis cohorts with mandatory loperamide and 38\% with mandatory colestipol/loperamide PRN) [38] as well as a higher mean cumulative dose of neratinib compared to ExteNET $(67,364.0 \mathrm{mg}$ vs. $54,193.9 \mathrm{mg})$ [31, 38, 39]. Notably, neratinib DE was effective for managing neratinib-induced diarrhea in the patient with $\mathrm{MBC}$ described in case 5 , consistent with the FDA-approved dosing strategy for MBC in addition to ESBC [31]. Because early-onset diarrhea may lead to neratinib discontinuation, proactive management of diarrhea with neratinib DE may allow for continuation of therapy across neratinib treatment settings.

Antidiarrheal prophylaxis with loperamide and colestipol was effective for managing diarrhea during the early course of treatment in case 4, although neratinib dose reduction was eventually required because of subsequent PPE. Prophylactic loperamide and colestipol reduced the incidence and severity of diarrhea in CONTROL, resulting in a diarrhea-related discontinuation rate of 4\%; however, as described above, prophylactic strategies were associated with a higher rate of GI adverse events compared to DE [38]. In addition, these regimens may be more inconvenient for patients because of the number of pills/tablets required per day.

Although neratinib DE was used in most of the cases described here, some escalation schedules differed from the DE schedules evaluated in CONTROL (Fig. 1) [38]. Several patients in this series initiated neratinib on longer or slower DE intervals as a precaution due to comorbidities or other considerations that could increase the likelihood of discontinuation, suggesting that extended DE strategies may be an option. As described in case 3, for patients with slower DE or other interruptions, oncologists may consider prolonging the course of therapy to make up for missed doses.

The patient cases described in this series suggest that neratinib-associated diarrhea is generally manageable, even in patients with potentially complicating comorbidities. In the extended adjuvant setting, neratinib has been shown to offer greater benefit in patients with hormone receptor-positive, lymph node-positive disease who were $\leq 1$ year from completion of trastuzumab, but patients with clinically significant GI comorbidities were excluded from 


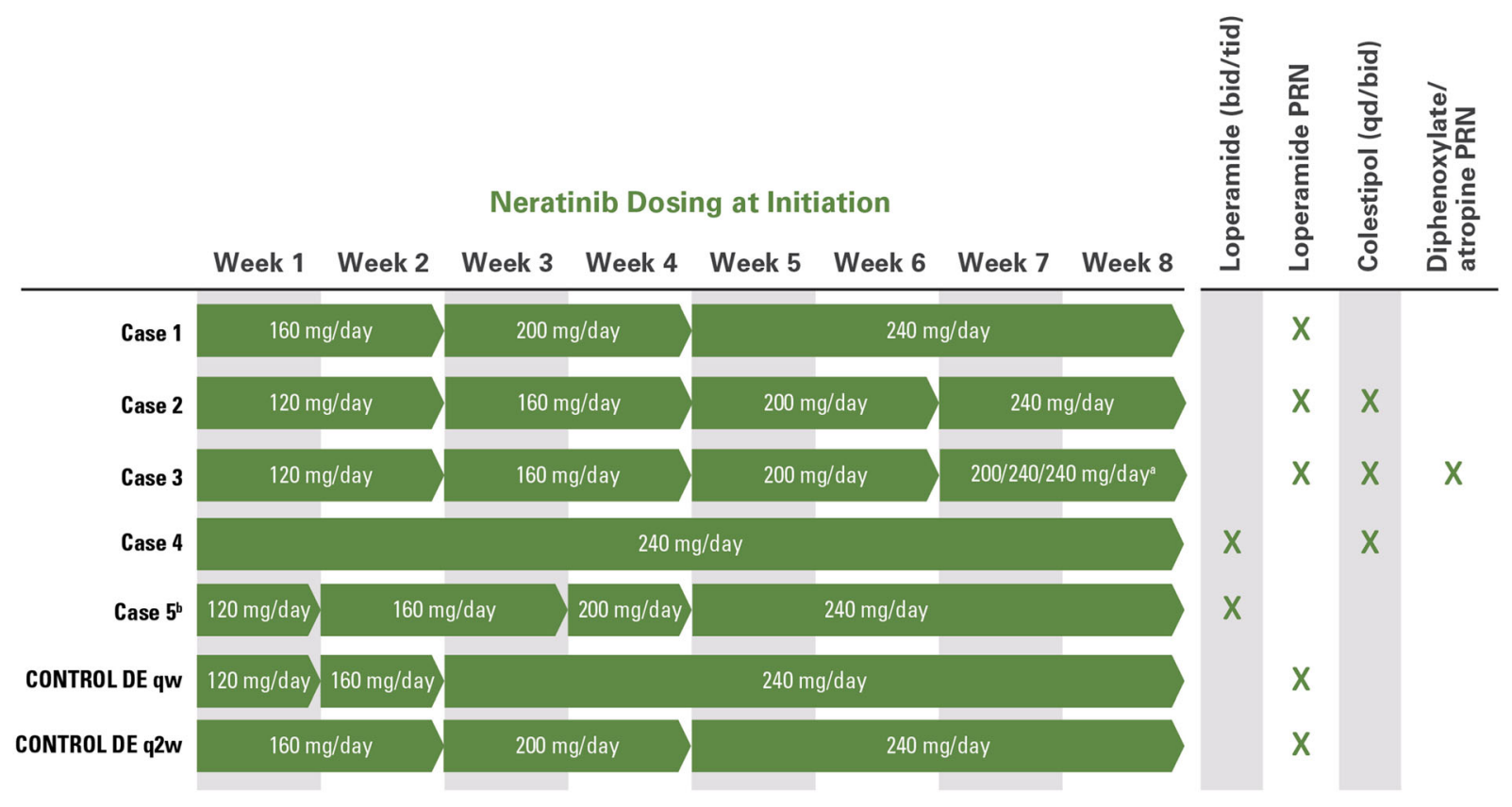

Fig. 1 Neratinib initiation strategies described in this case series. DE strategies evaluated in CONTROL are shown

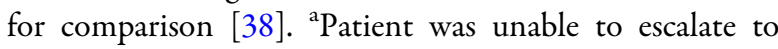
$240 \mathrm{mg} /$ day because of grade 2 to 3 diarrhea and instead maintained a 3-day repeating dose pattern. ${ }^{\mathrm{b}} \mathrm{In}$ combination with capecitabine $(1000 \mathrm{mg}$ bid). Patient reinitiated

ExteNET [12]. However, results reported in case 4 of this series demonstrate that a patient with well-managed Crohn's disease was able to complete the entire course of neratinib with implementation of prophylactic antidiarrheal treatment. Similarly, patients with HER $2+$ MBC who had previously been treated with an antiHER2 TKI were excluded from NALA [26]. The patient described in case 5 achieved disease response to neratinib plus capecitabine immediately following treatment with lapatinib plus capecitabine by managing neratinib-induced diarrhea and maintaining adherence to therapy using DE. These results suggest that some patients excluded from previous trials may be able to tolerate neratinib and thereby experience potential clinical benefit, meriting additional evaluation in an expanded patient population.

This case series highlights the importance of provider-patient communication in establishing patient expectations and managing adverse effects of neratinib. Early conversations may neratinib with $\mathrm{DE}$ after poor tolerability with a starting dose of $240 \mathrm{mg} /$ day and prophylactic loperamide. bid, twice daily; DE, dose escalation; PRN, as needed; qd, daily; qw, every week; q2w, every two weeks; tid, three times daily

help identify patient concerns and manage expectations about long-term therapy [41, 42]. As described in case 5, some patients may prefer the convenience of oral therapy, which is also a consideration during the COVID-19 pandemic to minimize in-person visits [43]. Likewise, reducing the incidence of severe diarrhea may decrease inpatient admissions or reduce the need for administering additional supportive care measures in a clinical setting, thereby decreasing contact with the health care system. Providing patients with a written plan during early consultations may alleviate anxiety and establish expectations for treatment [41], although the plan may change based on clinical factors. The patient described in case 2 was enrolled in a patient management program through a specialty pharmacy, which augmented educational counseling at neratinib initiation and provided additional support for managing adverse effects. As described in this case and in case 1 , frequent contact at initiation of neratinib may be beneficial, and pharmacists 
and advanced practice providers can play an important role in treatment optimization [44].

As described above, results from the CONTROL trial suggest that proactive diarrhea management with antidiarrheal prophylaxis or $\mathrm{DE}$ of neratinib from a lower starting dose can reduce the incidence, duration, and severity of neratinib-associated diarrhea in patients with early-stage breast cancer [38]. The 2-week DE schedule (week $1=120 \mathrm{mg} / \mathrm{day}$, week $2=160 \mathrm{mg} /$ day, week 3 and onwards: $240 \mathrm{mg}$ /day) has been included in the FDA label for both HER2+ ESBC and MBC since June 2021 [31]. An alternative DE schedule with a panel recommendation to start neratinib at a dose of $120 \mathrm{mg} /$ day with PRN loperamide and escalate the dose by $40 \mathrm{mg} /$ day each week until patients reach the recommended dose of $240 \mathrm{mg}$ /day of neratinib has also been published [45].

\section{CONCLUSIONS}

In summary, this case series shows that neratinib-associated diarrhea can be effectively managed with neratinib DE from a lower initial starting dose or prophylactic antidiarrheal medications in a real-world clinical setting. Widespread adoption of the strategies described here may allow more patients to adhere to neratinib therapy and thereby improve clinical outcomes for patients with HER2+ breast cancer treated with neratinib.

\section{ACKNOWLEDGEMENTS}

Funding. This study and the journal's Rapid Service Fee were funded by Puma Biotechnology, Inc., Los Angeles, CA, USA.

Medical Writing, Editorial, and Other Assistance. Writing and editorial support for this manuscript was funded by Puma Biotechnology, Inc., Los Angeles, CA, USA, and provided by Melanie Styers, PhD, Kristen Evaul, $\mathrm{PhD}$, and Michelle Kean, PhD, of Brightly Network, LLC.
Authorship. All named authors meet the International Committee of Medical Journal Editors (ICMJE) criteria for authorship for this article, take responsibility for the integrity of the work as a whole, and have given their approval for this version to be published.

Author Contributions. All authors contributed to the conception and design of this case series, collection and assembly of data, data analysis and interpretation, and manuscript writing and revision. All authors read and approved of the final manuscript.

Disclosures. Megan L. Kruse: Consulting or advisory role: Lilly Oncology, Eisai, Novartis Oncology, Immunomedics, Puma Biotechnology, Inc.; Research funding: Tempus. Irene M. Kang: Consulting or advisory role: Bristol Myers Squibb, Puma Biotechnology, Inc.; Speakers' Bureau: Puma Biotechnology, Inc.; Open Payments link: https://openpaymentsdata.cms. gov/physician/1328730. Nusayba A. Bagegni: Research funding: Sermonix Pharmaceuticals, Inc., Pfizer, AstraZeneca, Xcovery Holding Company, LLC, Novartis Pharmaceuticals Company; Daiichi Sankyo. W. Todd Howell: Employment: Hematology and Oncology Associates of Alabama. Halle C.F. Moore: Research funding: Puma Biotechnology, Inc., Daiichi Sankyo, Roche, AstraZeneca, Sermonix Pharmaceuticals, Inc. Cynthia M. Bedell: Employment: Texas Oncology PA; Consulting or advisory role: AstraZeneca, Daiichi Sankyo; Speakers' bureau: Genentech, SeaGen, AstraZeneca, Puma Biotechnology, Inc., Daiichi Sankyo. Christopher T. Stokoe: Employment: Texas Oncology PA; Leadership: Texas Oncology PA; Honoraria: Puma Biotechnology, Inc.; Consulting or advisory role: Puma Biotechnology, Inc.; Research funding: US Oncology.

Compliance with Ethics Guidelines. This article is based on previously conducted studies and anonymized case descriptions and does not contain any new studies with human participants or animals performed by any of the authors. 
Data Availability. Data sharing is not applicable to this article as no datasets were generated or analyzed during the current study.

Open Access. This article is licensed under a Creative Commons Attribution-NonCommercial 4.0 International License, which permits any non-commercial use, sharing, adaptation, distribution and reproduction in any medium or format, as long as you give appropriate credit to the original author(s) and the source, provide a link to the Creative Commons licence, and indicate if changes were made. The images or other third party material in this article are included in the article's Creative Commons licence, unless indicated otherwise in a credit line to the material. If material is not included in the article's Creative Commons licence and your intended use is not permitted by statutory regulation or exceeds the permitted use, you will need to obtain permission directly from the copyright holder. To view a copy of this licence, visit http://creativecommons.org/licenses/bync/4.0/.

\section{REFERENCES}

1. Loibl S, Gianni L. HER2-positive breast cancer. Lancet. 2017;389:2415-29.

2. Cameron D, Piccart-Gebhart MJ, Gelber RD, et al. 11 years' follow-up of trastuzumab after adjuvant chemotherapy in HER2-positive early breast cancer: final analysis of the HERceptin Adjuvant (HERA) trial. Lancet. 2017;389:1195-205.

3. Slamon D, Eiermann W, Robert N, et al. Ten year follow-up of BCIRG-006 comparing doxorubicin plus cyclophosphamide followed by docetaxel $(\mathrm{AC} \rightarrow \mathrm{T})$ with doxorubicin plus cyclophosphamide followed by docetaxel and trastuzumab $(\mathrm{AC} \rightarrow \mathrm{TH})$ with docetaxel, carboplatin and trastuzumab $(\mathrm{TCH})$ in HER2+ early. Cancer Res. 2016;76:S5-04.

4. Perez EA, Romond EH, Suman VJ, et al. Trastuzumab plus adjuvant chemotherapy for human epidermal growth factor receptor 2-positive breast cancer: planned joint analysis of overall survival from NSABP B-31 and NCCTG N9831. J Clin Oncol. 2014;32:3744-52.

5. Brufsky AM, Mayer M, Rugo HS, et al. Central nervous system metastases in patients with HER2- positive metastatic breast cancer: Incidence, treatment, and survival in patients from registHER. Clin Cancer Res. 2011;17:4834-43.

6. Olson EM, Najita JS, Sohl J, et al. Clinical outcomes and treatment practice patterns of patients with HER2-positive metastatic breast cancer in the posttrastuzumab era. Breast. 2013;22:525-31.

7. Gianni L, Eiermann W, Semiglazov V, et al. Neoadjuvant and adjuvant trastuzumab in patients with HER2-positive locally advanced breast cancer (NOAH): follow-up of a randomised controlled superiority trial with a parallel HER2-negative cohort. Lancet Oncol. 2014;15:640-7.

8. Gianni L, Pienkowski T, Im YH, et al. 5-year analysis of neoadjuvant pertuzumab and trastuzumab in patients with locally advanced, inflammatory, or early-stage HER2-positive breast cancer (NeoSphere): a multicentre, open-label, phase 2 randomised trial. Lancet Oncol. 2016;17:791-800.

9. Schneeweiss A, Chia S, Hickish T, et al. Long-term efficacy analysis of the randomised, phase II TRYPHAENA cardiac safety study: evaluating pertuzumab and trastuzumab plus standard neoadjuvant anthracycline-containing and anthracycline-free chemotherapy regimens in patients with HER2-positive ea. Eur J Cancer. 2018;89: 27-35.

10. Von Minckwitz G, Procter M, de Azambuja E, et al. Adjuvant pertuzumab and trastuzumab in early HER2-positive breast cancer. $N$ Engl J Med. 2017;377:122-31.

11. Von Minckwitz G, Huang CS, Mano MS, et al. Trastuzumab emtansine for residual invasive HER2positive breast cancer. N Engl J Med. 2019;380: 617-28.

12. Martin M, Holmes FA, Ejlertsen B, et al. Neratinib after trastuzumab-based adjuvant therapy in HER2positive breast cancer (ExteNET): 5-year analysis of a randomised, double-blind, placebo-controlled, phase 3 trial. Lancet Oncol. 2017;18:1688-700.

13. Chan A, Delaloge S, Holmes FA, et al. Neratinib after trastuzumab-based adjuvant therapy in patients with HER2-positive breast cancer (ExteNET): a multicentre, randomised, double-blind, placebo-controlled, phase 3 trial. Lancet Oncol. 2016;17:367-77.

14. Verma S, Miles D, Gianni L, et al. Trastuzumab emtansine for HER2-positive advanced breast cancer. N Engl J Med. 2012;367:1783-91.

15. Modi S, Saura C, Yamashita T, et al. Trastuzumab deruxtecan in previously treated HER2-positive breast cancer. N Engl J Med. 2020;382:610-21. 
16. Baselga J, Gelmon KA, Verma S, et al. Phase II trial of pertuzumab and trastuzumab in patients with human epidermal growth factor receptor 2-positive metastatic breast cancer that progressed during prior trastuzumab therapy. J Clin Oncol. 2010;28: 1138-44.

17. Corteś J, Fumoleau P, Bianchi GV, et al. Pertuzumab monotherapy after trastuzumab-based treatment and subsequent reintroduction of trastuzumab: activity and tolerability in patients with advanced human epidermal growth factor receptor 2-positive breast cancer. J Clin Oncol. 2012;30:1594-600.

18. Awada A, Colomer R, Inoue K, et al. Neratinib plus paclitaxel vs trastuzumab plus paclitaxel in previously untreated metastatic ERBB2-positive breast cancer the NEfERT-T randomized clinical trial. JAMA Oncol. 2016;2:1557-64.

19. Blackwell KL, Burstein HJ, Storniolo AM, et al. Overall survival benefit with lapatinib in combination with trastuzumab for patients with human epidermal growth factor receptor 2-positive metastatic breast cancer: final results from the EGF104900 study. J Clin Oncol. 2012;30:2585-92.

20. Blackwell KL, Burstein HJ, Storniolo AM, et al. Randomized study of lapatinib alone or in combination with trastuzumab in women with ErbB2positive, trastuzumab-refractory metastatic breast cancer. J Clin Oncol. 2010;28:1124-30.

21. Geyer CE, Forster J, Lindquist D, et al. Lapatinib plus capecitabine for HER2-positive advanced breast cancer. N Engl J Med. 2006;355:2733-43.

22. Cameron D, Casey M, Press M, et al. A phase III randomized comparison of lapatinib plus capecitabine versus capecitabine alone in women with advanced breast cancer that has progressed on trastuzumab: Updated efficacy and biomarker analyses. Breast Cancer Res Treat. 2008;112:533-43.

23. Jacobs SA, Robidoux A, Abraham J, et al. NSABP FB7: a phase II randomized neoadjuvant trial with paclitaxel + trastuzumab and/or neratinib followed by chemotherapy and postoperative trastuzumab in HER2+ breast cancer. Breast Cancer Res. 2019;21: 133.

24. Park JW, Liu MC, Yee D, et al. Adaptive randomization of neratinib in early breast cancer. N Engl J Med. 2016;375:11-22.

25. Murthy RK, Loi S, Okines A, et al. Tucatinib, trastuzumab, and capecitabine for HER2-positive metastatic breast cancer. N Engl J Med. 2020;382: 597-609.

26. Saura C, Oliveira M, Feng Y-H, et al. Neratinib plus capecitabine versus lapatinib plus capecitabine in
HER2-positive metastatic breast cancer previously treated with $\geq 2$ HER2-directed regimens: phase III NALA trial. J Clin Oncol. 2020;38:3138-49.

27. Freedman RA, Gelman RS, Anders CK, et al. TBCRC 022: a phase II trial of neratinib and capecitabine for patients with human epidermal growth factor receptor 2-positive breast cancer and brain metastases. J Clin Oncol. 2019;37:1081-9.

28. Freedman RA, Gelman RS, Wefel JS, et al. Translational breast cancer research consortium (TBCRC) 022: a phase II trial of neratinib for patients with human epidermal growth factor receptor 2-positive breast cancer and brain metastases. J Clin Oncol. 2016;34:945-52.

29. Petrelli F, Ghidini M, Lonati V, et al. The efficacy of lapatinib and capecitabine in HER-2 positive breast cancer with brain metastases: a systematic review and pooled analysis. Eur J Cancer. 2017;84:141-8.

30. Lin NU, Borges $\mathrm{V}$, Anders $\mathrm{C}$, et al. Intracranial efficacy and survival with tucatinib plus trastuzumab and capecitabine for previously treated HER2-positive breast cancer with brain metastases in the HER2CLIMB trial. J Clin Oncol. 2020;38:2610-9.

31. NERLYNX ${ }^{\circledR}$ (neratinib) [package insert]. Puma Biotechnology, Inc, Los Angeles, CA, 2021

32. National Comprehensive Cancer Network (NCCN). NCCN clinical practice guidelines in oncology: breast cancer version 8.2021. Published 13 September 2021. Available from: https://www.nccn. org/professionals/physician_gls/pdf/breast.pdf

33. National Comprehensive Cancer Network (NCCN). NCCN clinical practice guidelines in oncology: central nervous system cancers version 2.2021. Published 8 September 2021. Available from: https://www.nccn.org/professionals/physician_gls/ pdf/cns.pdf

34. Rugo HS, Di Palma JA, Tripathy D, et al. The characterization, management, and future considerations for ErbB-family TKI-associated diarrhea. Breast Cancer Res Treat. 2019;175:5-15.

35. Mortimer J, Di Palma J, Schmid K, Ye Y, Jahanzeb M. Patterns of occurrence and implications of neratinib-associated diarrhea in patients with HER2positive breast cancer: analyses from the randomized phase III ExteNET trial. Breast Cancer Res. 2019;21:32.

36. Gnant M, Iwata H, Bashford A. Duration of extended adjuvant therapy with neratinib in early-stage HER2 + breast cancer after trastuzumab-based therapy: exploratory analyses from the phase III ExteNET trial. J Clin Oncol. 2018;36:524. 
37. Moy B, Takahashi M, Ohtani S, Chmielowska E. Association between treatment duration and overall survival in early-stage HER2+ breast cancer patients receiving extended adjuvant therapy with neratinib in the ExteNET trial. J Clin Oncol. 2021;39:540.

38. Barcenas CH, Hurvitz SA, Di Palma JA, et al. Improved tolerability of neratinib in patients with HER2-positive early-stage breast cancer: the CONTROL trial. Ann Oncol. 2020;31:1223-30.

39. Marx G, Chien AJ, Garcia-Saenz J, Chan A. Dose escalation for mitigating diarrhea: ranked tolerability assessment of antidiarrheal regimens in patients receiving neratinib for early-stage breast cancer. J Clin Oncol. 2021;39:536.

40. Llovet P, Illana FJ, Martín-Morales L, et al. A novel TP53 germline inframe deletion identified in a Spanish series of Li-fraumeni syndrome suspected families. Fam Cancer. 2017;16:567-75.

41. Balogh EP, Ganz PA, Murphy SB, Nass SJ, Ferrell BR, Stovall E. Patient-centered cancer treatment planning: improving the quality of oncology care. Summary of an Institute of Medicine workshop. Oncologist. 2011;16:1800-5.

42. Untch M, Martin M, De Laurentiis M, Gligorov J. How to optimise extended adjuvant treatment with neratinib for patients with early HER2+ breast cancer. Oncol Ther. 2021. Online ahead of print. https://doi.org/10.1007/s40487-021-00153-5.

43. Sheng JY, Santa-Maria CA, Mangini N, et al. Management of breast cancer during the COVID-19 pandemic: a stage- and subtype-specific approach. JCO Oncol Pract. 2020;16:665-74.

44. Hicks KM, Cope D, Novak J, Scherer S. The role of the advanced practitioner in maintaining adherence in patients on oral oncolytics. J Adv Pract Oncol. 2017;8:7-21.

45. Jackisch $\mathrm{C}$, Barcenas $\mathrm{CH}$, Bartsch R, et al. Optimal strategies for successful initiation of neratinib in patients with HER2-positive breast cancer. Clin Breast Cancer. 2021;21:e575-83. 